
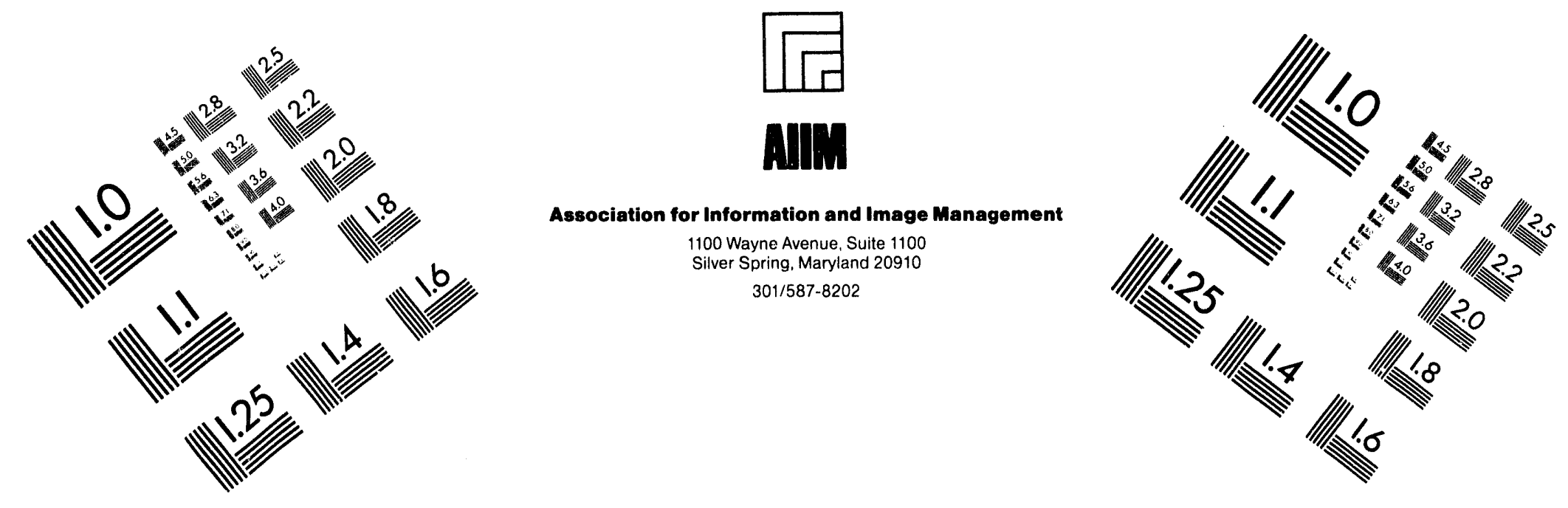

\title{
Centimeter
}

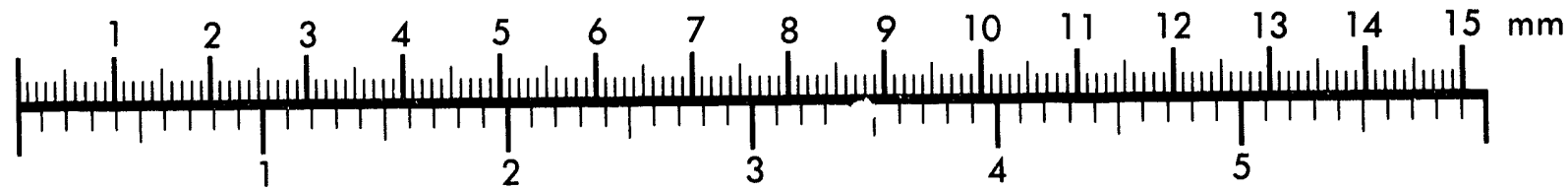

Inches
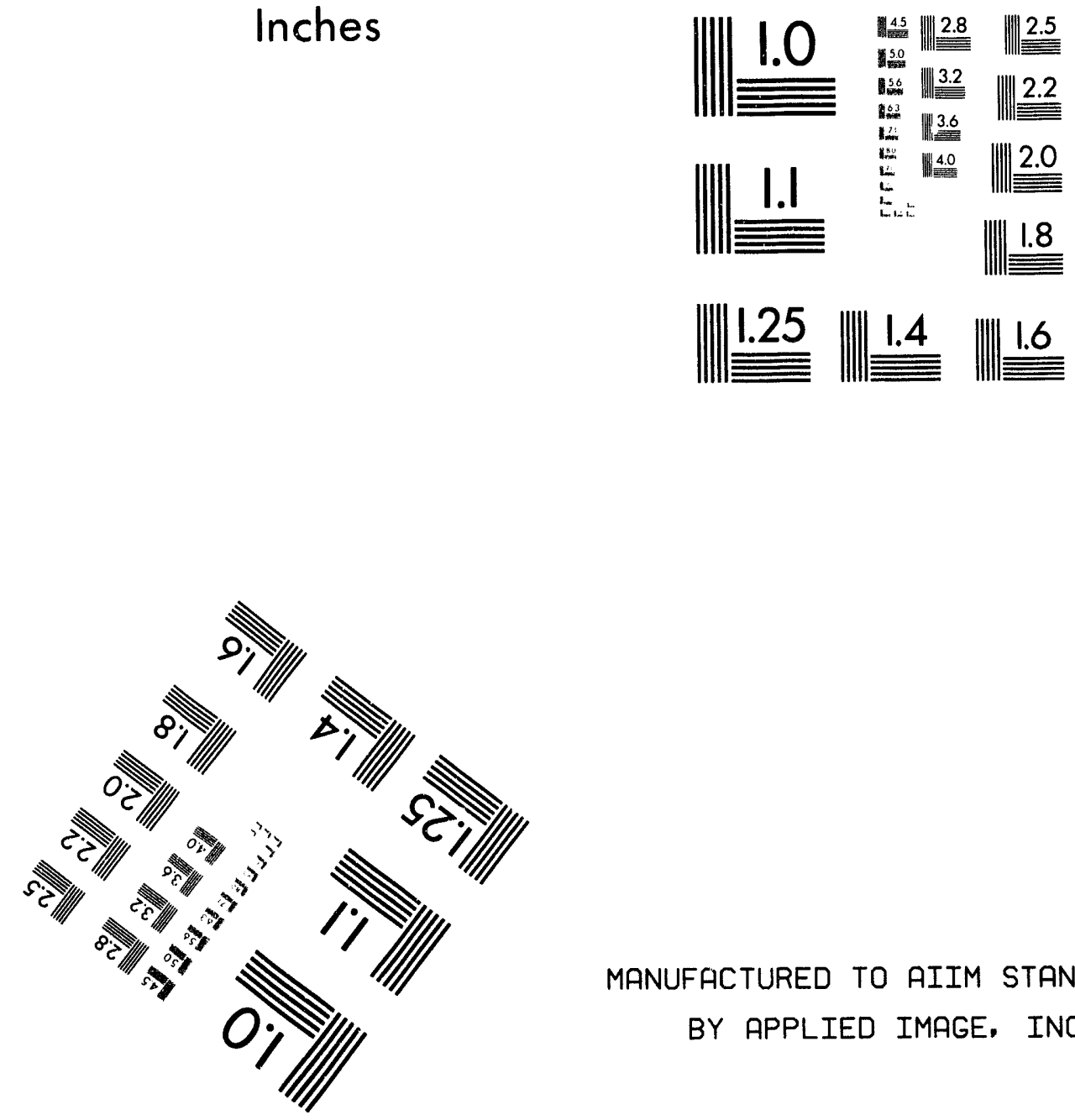

MANUFACTURED TO AIIM STANDARDS

BY APPLIED IMAGE, INC.

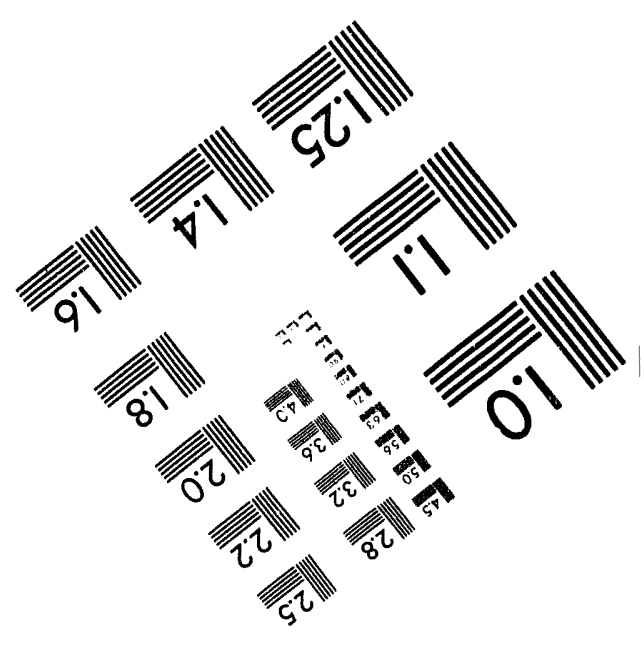



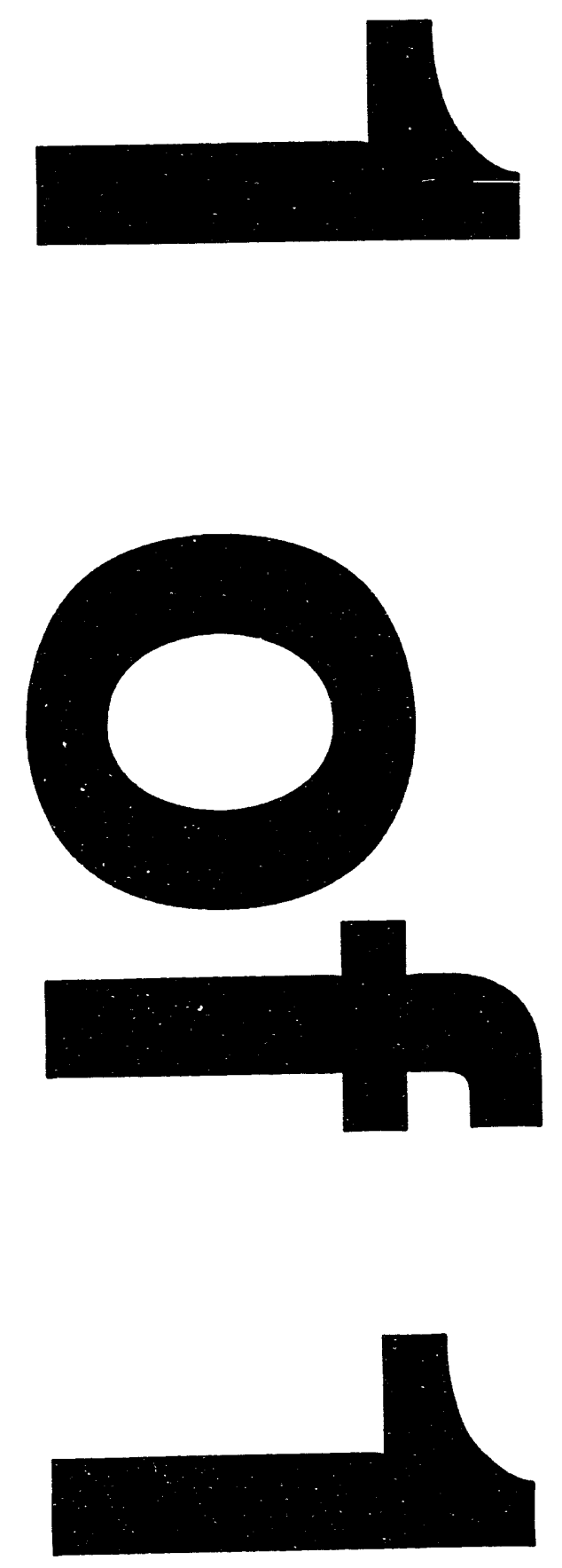


\title{
TOWARD A DESIGN FOR THE ITER PLASMA SHAPE AND STABILITY CONTROL SYSTEM
}

\author{
by \\ D.A. HUMPHREYS, R.H. BULMER, ${ }^{\star}$ S.W. HANEY, * A.G. KELLMAN, \\ J.A. LEUER, L.D. PEARLSTEIN, ${ }^{\star}$ and A. PORTONE ${ }^{\dagger}$
}

This is a preprint of a paper to be presented at the Eleventh Topical Meeting on the Technology of Fusion Energy (ANS), June 19-23, 1994, New Orleans, Louisiana, and to be printed in the Proceedings.

Work supported by

U.S. Department of Energy

Contract Nos. DE-AC03-89ER51114 and W-7405-ENG-48

*Lawrence Livermore National Laboratory

†ITER Joint Central Team

GENERAL ATOMICS PROJECT 3466

JULY 1994

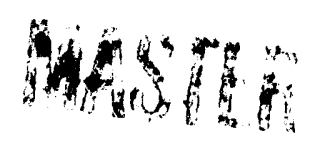




\section{TOWARD A DESIGN FOR THE ITER PLASMA SHAPE AND STABILITY CONTROL SYSTEM}

\author{
D.A. Humphreys, J.A. Leuer, \\ A.G. Kellman \\ General Atomics \\ P.O. Box 85608 \\ San Diego, CA 92186-9784 \\ (619) 455-2286
}

\author{
S.W. Haney, R.H. Bulmer, \\ L.D. Pearlstein \\ Lawrence Livermore \\ National Laboratory \\ P.O. Box 808 \\ Livermore, CA 94551-9900
}

\author{
A. Portone \\ ITER Joint Central Team \\ c/o Japan Atomic Energy \\ Research Institute (JAERI) \\ Naka-machi, Naka-gun \\ Ibaraki-ken \\ 311-01 Japan
}

\begin{abstract}
A design strategy for an integrated shaping and stability control algorithm for ITER is described. This stategy exploits the natural multivariable nature of the system so that all poloidal field coils are used to simultaneously control all regulated plasma shape and position parameters. A nonrigid, flux-conserving, linearized plasma response model is derived using a variational procedure analogous to the ideal MHD Extended Energy Principle. Initial results are presented for the nonrigid plasma response model approach applied to an example DIII-D equilibrium. For this example, the nonrigid model is found to yield a higher passive growth rate than a rigid current-conserving plasma response model. Multivariable robust controller design methods are discussed and shown to be appropriate for the ITER shape control problem.
\end{abstract}

\section{INTRODUCTION}

An important component of the ITER Engineering Design Activity (EDA) is an initial design of the plasma shaping and stability control system. In general, the necessary control accuracies and precisions for ITER are comparable (as fractions of the minor radius) to those achieved in present-day experiments. However, the number of poloidal field (PF) coils available for shaping control in ITER is smaller than the number available in most highly shaped tokamak plasma experiments. These coils are also more distant from the plasma (relative to the minor radius) than is typical in other tokamaks. The ability of the PF coils to affect the plasma shape is therefore severely limited, making optimization of the control algorithm both crucial and difficult.

In present-day shaped tokamaks, the typical vertical instability growth time is much shorter than the typical plasma shaping response time, allowing shape and vertical stability control to be analyzed and accomplished independently of each other. By contrast, ITER plasma shaping and vertical stability response times are comparable, owing to the low elongation and strong coupling between plasma and conducting structures. In such a case, an integrated stability and shaping control algorithm can be most efficiently designed using multivariable control analysis and design techniques. These methods can take advantage of the similar shaping and stability time scales and make efficient use of the limited control degrees of freedom.

The control philosophy guiding the design involves the use of "robust" design techniques and control law "gain scheduling." Gain scheduling involves determining control laws for a finite set of operating point equilibria and changing the controller specification dynamically as the shot evolves. The controller selected at any given time during the shot will depend on the plasma kinetic and current profile state as well as the phase the shot is in. Robust control design and analysis techniques seek to ensure adequate performance in the presence of expected levels of noise, disturbance, and model error.

The design procedure involves determination of the operating point equilibria, calculation of the 
perturbational plasma responses about the operating point equilibria, design of a robust controller, and both linear and nonlinear tim -cependent simulation of the closed-loop system behavior.

Section II describes features of the ITER control problem and specifications by the ITER Joint Central Team (JCT). The general tokamak stability and shaping control problem is formulated in Section III. Plasma response models required for tokamak control design are discussed in Section IV. A nonrigid flux-conserving model is derived and compared with a current-conserving rigid vertical shift plasma model. Section V discusses the advantages of robust design in the tokamak equilibrium control problem, and Section VI contains summary comments and discussion.

\section{ITER CONTROL CHARACTERISTICS}

\section{A. Present ITER Baseline Design}

A recent form of the ITER EDA baseline machine geometry is shown in Fig. 1. The figure illustrates the lower single-null plasma configuration and the relatively small number of PF coils available for shaping. The most recent design includes a $25 \mathrm{MA}$ toroidal plasma current, $8.1 \mathrm{~m}$ major radius, $3.0 \mathrm{~m}$ minor radius, $\kappa_{95}=1.54$ elongation, and a $5.7 \mathrm{~T}$ toroidal field on axis. The nominal passive growth rate for a passive structure with total toroidal resistance of $10 \mu \Omega$ is approximately $1.5 \mathrm{~s}^{-1} .^{1}$ As is often the case for tokamak vertical stability control, this growth time is comparable to the magnetic diffusion time through the conducting structure. However, ITER is unusual in that other plasma shape response modes have damping rates which are also comparable to this diffusion time. This means that control algorithms for both plasma shaping and stability control must take the overall system dynamic response into account and compensate properly for the resulting phase lags.

\section{B. Control Conventions and Assumptions}

The basis for the design of the ITER plasma control system was broadly determined by the 2nd ITER Technical Meeting on Plasma Equilibrium \& Control (Naka Joint Work Site, November 1993). This meeting along with further determinations by the ITER JCT specified the conventions to be followed by the Member Home Teams in the design of the equilibrium control system. The principal

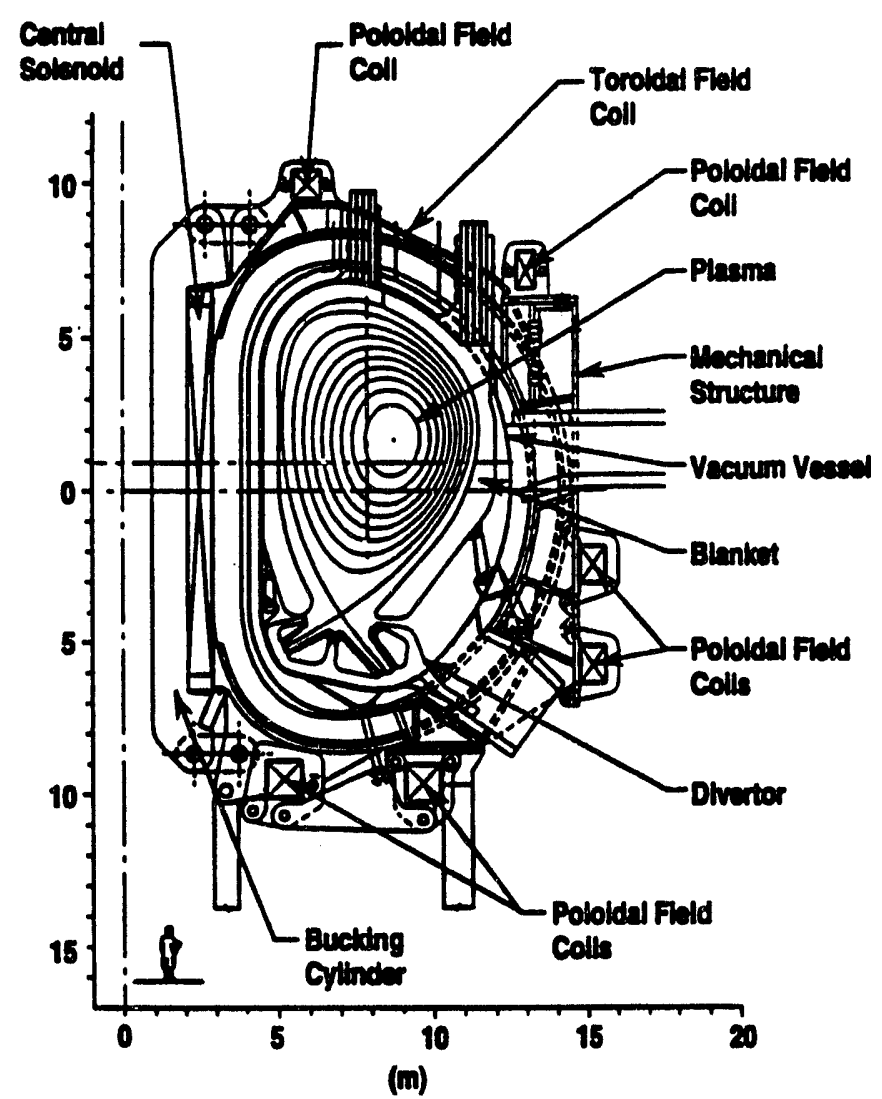

Fig. 1. ITER TAC-4 baseline geometry. The figure illustrates the lower single-null plasma configuration and the relatively small number of poloidal field (PF) coils available for shaping.

assumption is that six of the seven superconducting PF coils are to be simultaneously available for shaping and stability control. No subset of these six coils is to be dedicated to control of any particular equilibrium shape parameter, such as vertical position. The remaining (inboard ohmic) coil will be dedicated to plasma current control.

Guidance was also provided by the JCT on the set of plasma parameters to be be controlled in ITER. Seven parameters can be controlled in principle with the seven PF coils provided in the present EDA design. The chosen set of controlled parameters is comprised of the plasma current and six gaps between the plasma separatrix and the neighboring first wall surface. A schematic illustration of the control gaps is given in Fig. 2. Two of these gaps (G1, G2) define the distance between separatrix and divertor throat wall. Two of the gaps outside the divertor region must reflect the plasma-antenna distance in the extreme outboard 


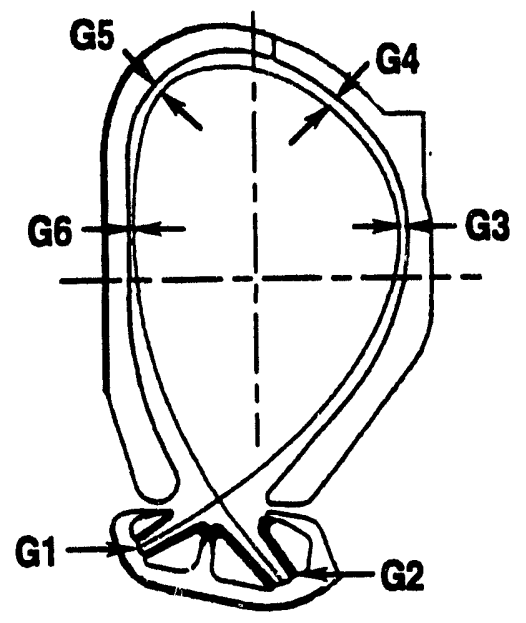

Fig. 2. ITER gap control geometry.

region (G3) and the gap in the upper-outboard high toroidal field ripple region (G4). The remaining two gaps define the distance between plasma and points on the upper-inboard (G5) and extreme inboard (G6) walls. The exact position of the four gaps outside the divertor region may be allowed to vary as the discharge evolves in order to optimize control performance.

For design purposes it is being assumed that full knowledge of the plasma state (gap spacings) and some estimate of conductor eddy currents is available from an appropriate diagnostic array and a real time equilibrium reconstruction tool.

\section{TOKAMAK SHAPE AND STABILITY CONTROL}

The shape of a tokamak plasma is essentially determined by the axisymmetric toroidal currents flowing in the conducting structure and PF coils, along with certain internal plasma characteristics. For modeling purposes, large continuous conducting structures such as the vacuum vessel are divided into arrays of discrete current paths, each of whose currents contribute an entry to a vector $I_{s}$. Along with the set of internal plasma quantities, $I_{s}$ completely describes the state of the system.

The internal plasma quantities include the plasma current, $I_{p}$, the confinement efficiency parameter, $\beta_{p}$, and the normalized internal inductance per unit length, $\ell_{i}$. These quantities are used as a basis for "gain scheduling:" when their values depart sufficiently from the specified operating point, a new set of control gains optimized for the new regime must be used. The plasma current is expected to be regulated by a separate control loop which drives the ohmic coil alone. However, appropriately modified control laws must be gain scheduled as well for circumstances under which the plasma current departs significantly from the set point.

The evolution of currents in conductors (and thereby the plasma state) is described by a set of circuit equations, derived from Faraday's Law, which include the plasma response to current variations. The circuit equations can be written as a first order linear matrix differential equation:

$$
\mathbf{M}_{s s} \dot{I}_{s}+\mathbf{R}_{s s} I_{s}+\mathbf{X}_{s s} \dot{I}_{s}+=\mathbf{B}_{1} V_{p f}
$$

where subscript " $s$ " refers to the complete set of stabilizing conductors and $\dot{I}_{s}$ represents the time derivative of the conductor current vector. The vector $V_{p f}$ denotes the control voltages applied to active $\mathrm{PF}$ coils. The non-square matrix $\mathbf{B}_{1}$ contains an identity matrix of rank equal to the number of PF coils in its upper portion, and all zero entries in its lower portion. The upper portion ide.1tity matrix allows active voltage to be applied to PF coils alone, while the lower portion zero matrix reflects the zero voltage of passive (e.g. vessel) conductor elements. $\mathbf{M}_{s s}$ is the conductor mutual inductance matrix, and $\mathbf{R}_{s s}$ is the conductor resistance matrix. The plasma response matrix $\mathbf{X}_{s s}$ describes flux variations at the conductors due to plasma variations which in turn arise from conductor current changes. $\mathbf{X}_{s s}$ therefore completely specifies the linearized plasma response to conductor current state variables. Conductor variations described by this matrix include changes in plasma current which must occur on the fast time scale to conserve flux, although the operating point plasma current is taken to be fixed by a separate control loop not described in these equations.

The circuit equation, Eq. (1), can be readily cast in the "state space" form with simple matrix manipulations once the geometry-dependent and plasma response terms have been determined. The state space representation of a dynamic system described by a set of coupled linear first order differential equations consists of a first-order matrix "state" equation and a matrix "output" equation. The state equation describes the time evolution of the system's state variables while the output equation Jescribes the dependence of sensed quantities on state and control variables. For the shape control problem we have described, the state space can 
be written as

$$
\begin{gathered}
\dot{I}_{s}=\mathbf{A} I_{s}+\mathbf{B} V_{p f} \\
y=\mathbf{C} I_{s}+\mathrm{D} V_{p f}
\end{gathered}
$$

where the conductor current vector $I_{s}$ is now known as the "state" vector, and consists of perturbations in PF coil and vessel currents from their equilibrium values. The $\mathrm{PF}$ coil voltage vector $V_{p f}$ is now referred to as the "control" vector, and similarly corresponds to perturbations in PF voltages from equilibrium values. The sensed variable vector $y$ typically consists of perturbations from equilibrium values in magnetic field probe, flux loop, and Rogowski loop signals for the general tokamak control problem. However, because of the knowledge of the plasma state to be assumed in the present design effort (see Section I), the sensed variable vector consists of gap error values, measured perturbed PF coil currents, and estimated perturbed passive conductor currents. In what follows, system vectors will be implicitly understood to contain perturbed quantities only.

Model error must produce some degree of uncertainty in all of these signals. However, an extra uncertainty must be ascribed to the passive conductor currents, as these are to be estimated from magnetic signals in the final realization of the control design.

\section{PLASMA RESPONSE MODEL}

\section{A. Operating Point Equilibria}

Let the term "control phase" denote a time interval during which a single control law is expected to be used to maintain stable plasma evolution. A single shot evolution process, such as plasma current rampup, might include several control phases, each of which will require its own control law. An entire shot might therefore contain many such control phases.

During a control phase, the plasma may be expected to evolve in some way or to remain in a steady state. In either case, a single operating point equilibrium must be defined to represent each control phase to be expected during the evolution of a shot, and a single nominal control law must be found for each phase. For a control phase containing expected plasma variation (e.g. rampup or profile relaxation following the removal of beam heating), the robustness of the nominal control law throughout the phase must be demonstrated a posteriori.

A sufficient number of control laws must also be determined to account for the entire range of possible though unpredictable variations in internal plasma quantities during each control phase.

\section{B. Nonrigid Model Derivation}

In order to design a nominal control law for a particular choice of equilibrium, a plasma response model is required. For the equilibrium control problem, such a model fundamentally must describe the variation in flux at the conductors as well as the influence of the plasma on the controlled parameters. These correspond to the matrix $\mathbf{X}_{s s}$ in Eq. (1) and the plasma contribution to the output matrix $\mathbf{C}$ in Eq. (2).

In this section we describe a plasma response model which is computed using a vertical stability package integrated with the TEQ free-boundary MHD equilibrium code. The plasma is treated as an ideal MHD fluid and the conducting plates and coils surrounding the plasma are decomposed into a series of resistive, axisymmetric toroidal rings. The characteristic timescale of plasma motion is assumed to be much slower than ideal MHD growth times (i.e., plasma inertia is ordered small). This is equivalent to assuming the plasma to be in equilibrium at all times on the timescale of the plasma response of interest. Under these circumstances, the growth rate of any instability is dependent on the component of the plasma displacement perpendicular to the flux surfaces and the currents induced or driven in the conductors surrounding the plasma.

The perpendicular displacement is solved for using a variational procedure analogous to the Extended Energy Principle of ideal MHD: an expression representing the potential energy for the plasma and vacuum regions is minimized with respect to trial functions for the displacement. ${ }^{2} \mathrm{~A}$ matrix equation of the form ${ }^{3}$

$$
\left(\mathbf{W}_{F}+\mathbf{W}_{V}\right) \xi+\mathbf{W}_{s} I_{s}=0
$$

is obtained, where the matrices $\mathbf{W}_{F}$ and $\mathbf{W}_{V}$ represent the plasma and vacuum energies respectively, the matrix $\mathbf{W}_{s}$ represents the effect of conductors, the vector $\xi$ represents the displacement trial functions, and the vector $I_{s}$ represents conductor currents as in Eqs. (1) and (2). The displacement and the conductor currents are also related by a matrix 
circuit equation similar to Eq. (1):

$$
\mathbf{P} \dot{\xi}+\left(\mathbf{M}_{s s}+\mathbf{M}_{\text {corr }}\right) \dot{I}_{s}+\mathbf{R}_{s s} I_{s}=\mathbf{B}_{1} V_{p f}
$$

where $\mathbf{P}$ represents the plasma-conductor inductance due to plasma deformation and is related to $\mathbf{W}_{\text {s. }} \quad \mathbf{M}_{\text {corr }}$, also arising from the Energy Principle calculation, represents the correction to the conductor mutual inductance matrix due to image currents in the plasma. All other quantities are as defined previously. These two sets of matrix equations can be solved simultaneously to yield the displacements, currents, and growth rates of the system's normal modes. Combining Eqs. (3) and (4) and comparing the result with Eq. (1), the plasma response matrix is found to be $\mathbf{X}_{s s} \equiv$ $\mathbf{P}\left(\mathbf{W}_{F}+\mathbf{W}_{V}\right)^{-1} \mathbf{W}_{s}+\mathbf{M}_{\text {corr }}$.

The plasma contribution to the $\mathbf{C}$ matrix can be determined in a similar way, calculating the effect of the plasma on controlled variables instead of its effect on conductor flux.

\section{Comparison of Rigid and Nonrigid Models}

The most common plasma response model used in the analysis of tokamak vertical stability assumes a current-conserving, rigid shift of the plasma current distribution for the displacement. While not consistent with the ideal MHD model, such a plasma response can be easily determined from an equilibrium calculation and lends itself readily to simple circuit a alysis. It is also the correct limit for a filamentary (infinite inductance) plasma, a common chcice for the lowest order approximation to the actual instability. In what follows, "rigid" will always refer to the currentconserving rigid vertical displacement model, and "nonrigid" will refer to the flux-conserving nonrigid plasma response model.

Because the plasma can only move rigidly and vertically in the rigid model, only one distribution of flux perturbation among conductors is produced by plasma motion. That is, the resulting plasma response matrix, $\mathbf{X}_{s s}$ is unity rank, and produces only a single flux perturbation vector at the conductors (of varying amplitude) regardless of the conductor current vector. As an example, we use the lower single-null DIII-D equilibrium whose flux contours (level values in Wb) are shown in Fig. 3 along with DIII-D machine geometry. For this example, only vacuum vessel elements are used for stabilization.

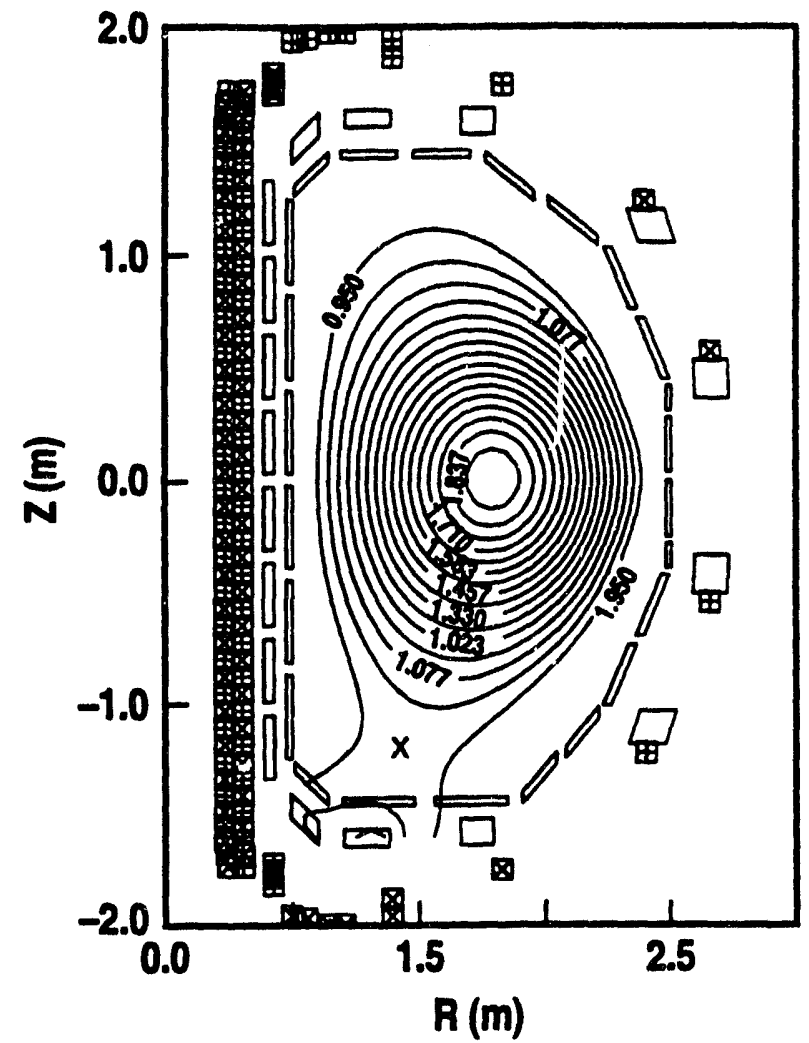

Fig. 3. DIII-D geometry with flux contours (levels in Wb) for a lower single-null diverted plasma. An " $x$ " drawn immediately below the last closed flux surface denotes the $X$-point location.

Flux perturbations due to plasma alone corresponding to the rigid current-conserving vertical shift are illustrated in Fig. 4. This mode shape corresponds to any column (or row) of the (unity rank) $\mathbf{X}_{s s}$ matrix for the rigid vertical shift. The open circles represent negative flux perturbation at the stabilizing vessel elements, while the " + " symbols represent positive flux perturbations. The radius of each circle is proportional to the amplitude of the perturbation at that conductor. The dotted lines are contours of flux which would result if the mode consisted of currents in the vessel. These contour lines are meant only to provide another means of judging the symmetries $c f$ the mode. The mode shown is clearly related to a vertical, largely updown symmetric displacement of the plasma.

By comparison, the nonrigid plasma response matrix can exhibit several significant modes of flux perturbation at the vessel. Performing a singular value decomposition (SVD) of the nonrigid $\mathbf{X}_{s s}$ 


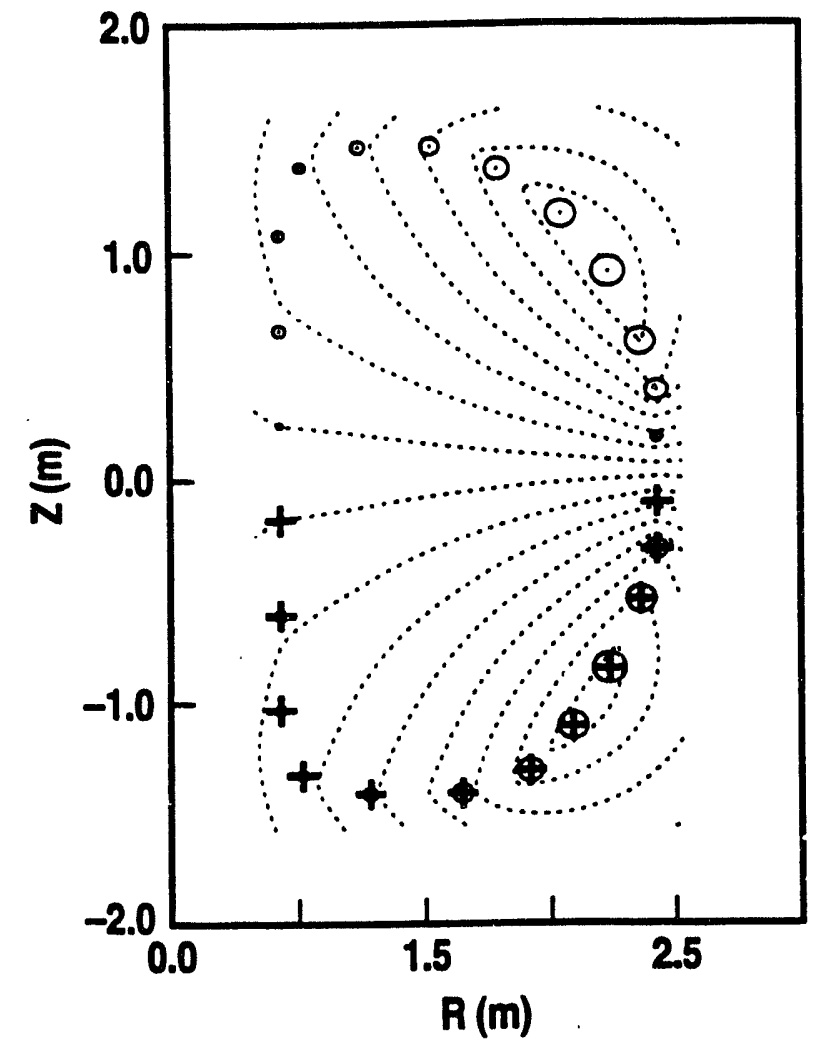

Fig. 4. Change of flux at conductors due to plasma displacement and current variation alone. The response shown results from a constant current, rigid, vertical plasma displacement.

matrix extracts the principal axes, or modes of the plasma response. Defining this SVD by $\mathbf{X}_{s s}=$ $\mathbf{U} \boldsymbol{\Sigma} \mathbf{V}^{\mathbf{T}}$, the plasma response principal axes are the columns of the $U$ matrix. The mode associated with the largest singular value is shown in Fig. 5 with the same symbology as in the previous figure. As expected, this dominant mode is very close to the rigid vertical plasma displacement shown in Fig. 4, but involves non-vertical motion as well and is not as up-down symmetric as the rigid mode.

The mode associated with the second largest singular value represents a largely radial shift of the plasma and is shown in Fig. 6. Just as the "vertical" mode included non-vertical plasma motion, this "radial" mode clearly includes vertical displacements as well. While the actual plasmaconductor mode related to this plasma displacement mode is stable, the coupling to vertical motion means that its dynamics must be taken into account in a multivariable control design.

A comparison of the vertical instability growth rates arising from the rigid and nonrigid models

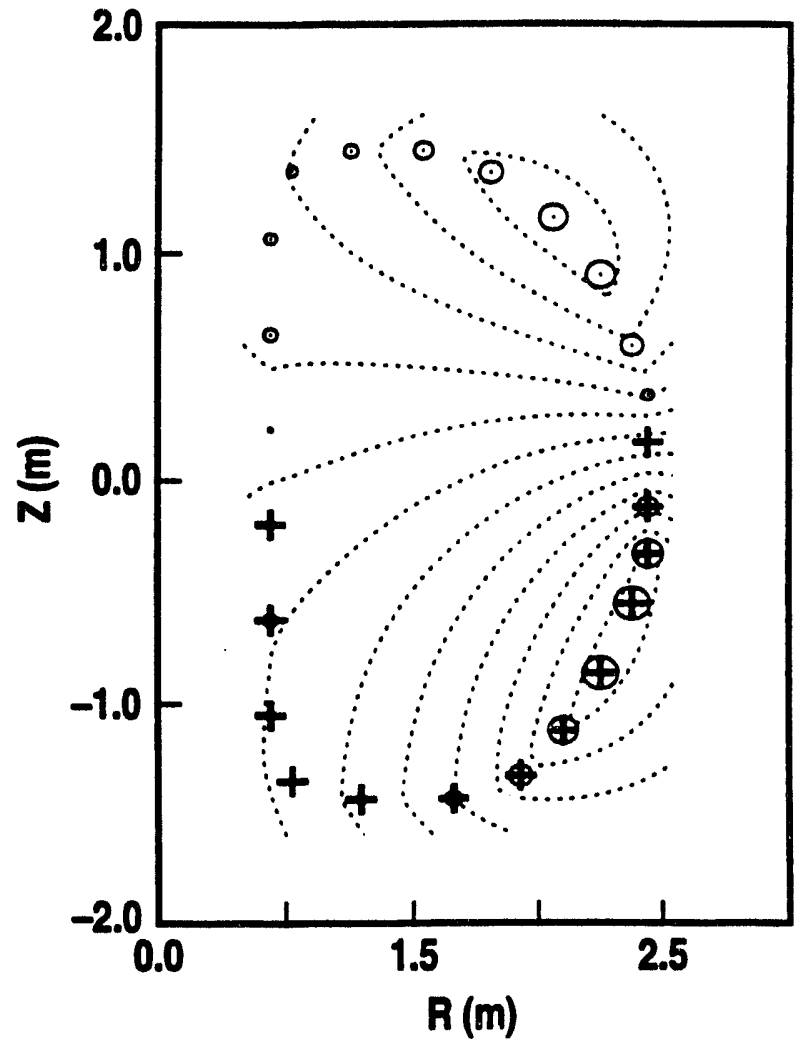

Fig. 5. Change of flux at conductors due to plasma displacement and current variation alone. The response shown results from a flux conserving, nonrigid, "vertical" mode plasma displacement.

also serves to illustrate the importance of using a nonrigid model. With only the vacuum vessel for passive stabilization, the rigid model produces a single unstable open loop mode with a growth rate of $\gamma_{r i g}=260 \mathrm{~s}^{-1}$. The nonrigid model similarly produces a single unstable open loop mode, but with a growth rate of $\gamma_{\text {non }}=315 \mathrm{~s}^{-1}$. This $20 \%$ difference is sufficient to produce unsatisfactory performance for one model using a controller designed with the other model.

It is interesting to note that in this case the rigid model growth rate was found to be less than that found for the nonrigid model. This ordering agrees with experimental results for tokamaks such as DIII-D and Alcator C-MOD ${ }^{4}$ as well as with theoretical analysis of the problem. ${ }^{5}$ However, some have found the opposite ordering in the case of ITER. ${ }^{6}$ The reasons for this are as yet unclear, but the present uncertainty serves to emphasize the need for a robust design which can ensure satisfactory performance for both models. 


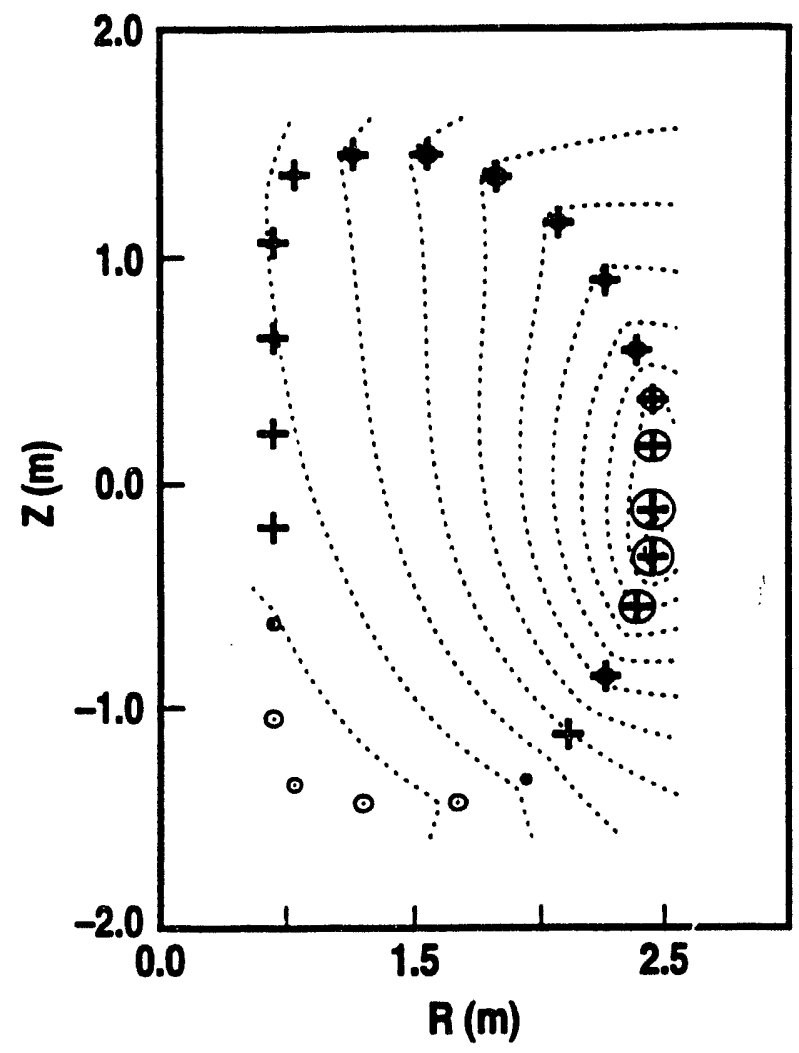

Fig. 6. Change of flux at conductors due to plasma displacement and current variation alone. The response shown results from a flux conserving, nonrigid, "radial" mode plasma displacement.

\section{MULTIVARIABLE ROBUST CONTROL DESIGN}

In studies on DIII-D, ${ }^{7} \mathrm{JT}-60 \mathrm{U},{ }^{8}$ and Alcator $\mathrm{C}-\mathrm{MOD},{ }^{4}$ it has not been possible to verify agreement between rigid filament or nonrigid MHD models and experiment below a 20-50\% accuracy level. For both kinds of plasma model, uncertainty in the conductor models and lack of knowledge of the equilibrium profiles has contributed to this level of agreement in all of the machine studies. The key point is that it is precisely this lack of knowledge of the equilibrium which faces the control designer: in actual machine operation precise knowledge of the profiles is in general not possible prior to achievement of the equilibrium.

This degree of uncertainty in plasma response can easily give rise to unsatisfactory performance in the final realization of a control system. A closedloop system which is underdriven as a consequence of model error can result in sluggish response or instability. An over-driven closed loop system can exhibit unacceptably high overshoot, long settling time, or even driven instability for an actual plasma. Failure to account for the actual noise and disturbance environment can also lead to unsatisfactory performance.

While some degree of improvement in conductor model accuracy is possible by experimental measurement of its dynamic response to coil excitation, the plasma response cannot always be safely measured before a working control system is needed. This is particularly true for a device such as ITER which can tolerate very few disruptions resulting from plasma control failure. In effect, the control system must function satisfactorily from the moment the machine is first operated in a highperformance mode. Furthermore, the evolution of profiles which strongly affect plasma dynamic response cannot be easily predicted in the complex heating, fueling, and burning environment of ITER. For such a device it is prudent to build some degree of robustness into the controller design from the outset.

A robust control design is one which provides satisfactory performance and stability even in the presence of model error, noise, or disturbances. Typically, a controller can be designed to be robust to one or two of these phenomena but not to all. For example, the requirements for insensitivity to noise are typically not compatible with the requirements for insensitivity to disturbances. Designers must choose a balance of robustness to each phenomenon which is appropriate for the system, making tradeoffs as necessary to ensure satisfactory performance. Such a balance can be achieved in multivariable systems using the robust control methods of $H_{\infty}$ design and structured singular value analysis ( $\mu$-analysis) and synthesis. ${ }^{9,10}$

This approach requires a degree of system specification not required by nonrobust techniques. The uncertainties expected in plasma response, conductor coupling, and power supply models must be characterized and incorporated into the nominal system model. Closed-loop performance criteria must be specified in terms of desired frequency responses. Robust design methods have the advantage, however, that excellent controllers can be designed even if knowledge of the actual plasma, conductor, and power supply responses is imperfect, as is the case for the tokamak plasma control problem. If a robust design solution can be found, closed loop stability will be guuranteed even with such imperfect knowledge of the system to be 
controlled. Precedent exists in the application of these methods to the problem of plasma vertical stabilization for the ITER Conceptual Design Activity (CDA) configuration. ${ }^{11}$

The procedure used to design a robust multivariable plasma controller is to:

1. Construct a generalized system model consisting of the physical system model, uncertainty models, and performance specification weighting functions:

a. Model the physical system to be controlled.

b. Add uncertainty models to specify robustness.

c. Add performance models to specify the required performance in terms of frequency dependent weighting of certain (typically error) signals in the physical system model.

2. Design the controller with $H_{\infty}$ design techniques.

3. Analyze robustness and performance of the closed loop system with Structured Singular Value $(\mu)$ analysis and both linear and nonlinear simulation.

Steps 2 and 3 are iterated until a suitable controller is found.

Robust control is a very conservative design approach because it attempts to design a controller for the worst case, least accurate model within the prespecified uncertainty and the worst external disturbances and/or noise level. Conservative approaches have both advantages and disadvantages. The principal advantage is that this approach maximizes confidence that when implemented, the design will perform satisfactorily (providing a sufficiently robust solution was found). The disadvantage is that $H_{\infty}$ and robust methods may not produce the absolute best design which optimizes costperformance criteria (because it effectively designs for the worst case, which seldom occurs).

Better response characteristics can be produced and cost-performance can be improved by employing less conservative design assumptions. However, the relaxation in uncertainty constraints must be warrented by some actual improved knowledge of the machine geometry or plasma response, which is not likely to be available until after ITER construction is complete. In the absence of such explicit improvement in model accuracy, robust techniques which take into account the actual expected uncertainty will provide maximum confidence in the achievability of reliable equilibrium control.

\section{SUMMARY AND DISCUSSION}

An approach to design of the shape and stability control system for ITER has been proposed which exploits multivariable robust control analysis and synthesis techniques. This design philosophy can produce controllers which provide satisfactory performance in the presence of expected levels of model error, noise, and disturbances. Plasma current, poloidal $\beta$, and internal inductance are used as bases for gain scheduling, in which new gain laws are chosen as these quantities vary.

An algorithm has been developed for computing nonrigid flux-conserving plasma response models appropriate for shaping and stability control design. The algorithm has been implemented in a package integrated with the TEQ free-boundary MHD equilibrium code. The plasma stability response package and TEQ together comprise a powerful tool contributing to the analysis and design of tokamak equilibrium control systems.

A comparison between nonrigid (fluxconserving) and rigid (current-conserving) plasma models demonstrated the difference in plasma motion allowed and the corresponding growth rates calculated for these models. For an example DIII-D equilibrium, the nonrigid model growth rate was found to exceed the rigid model growth rate by approximately $20 \%$.

Nonrigid ideal MHD plasma response models such as that described here are theoretically the most physically correct presently available, and include significant plasma degrees of freedom not represented in simple rigid plasma displacement models. These degrees of freedom must be taken into account in a multivariable equilibrium control design. Such models will allow design of an integrated shape and stability control system which will simultaneously drive six PF coils in ITER to regulate six plasma-wall gaps.

\section{ACKNOWLEDGMENT}

The authors would like to thank M.L. Walker for generous contributions of expertise in robust control methods.

This is a report of work supported by the U.S. Department of Energy under Contract Nos. DEAC03-89ER51114 and W-7405-ENG-48. 


\section{REFERENCES}

1 "Detail of the ITER Outline Design Report," Fourth Meeting of the Technical Advisory Committee (TAC-4), January 1994.

2 J.P. FREIDBERG, Ideal Magnetohydrodynamics, Plenum Press, (1987).

3 S.W. HANEY, "Methods for the Design and Optimization of Shaped Tokamaks," PFC Report PFC/RR-88-12, Massachusetts Institute of Technology, Cambridge, (1988).

4 G. TINIOS, Private Communication, April 1994.

5 I.H. HUTCHINSON, "Simplified Models of Axisymmetric Instabilities," Nucl. Fusion 29, 2107 (1989).

6 ITER Joint Central Team, Proc. of 2nd ITER Technical Meeting on Plasma Equilibrium and Control, ITER Naka Joint Work Site, November 1993 .

7 J.B. LISTER, E.A. LAZARUS, et al., "Experimental Study of the Vertical Stability of High Decay Index Plasmas in the DIII-D Tokamak," Nucl. Fusion 30, 2349 (1990).
8 D.A. HUMPHREYS, and R. YOSHINO, "JT60 U Vertical Stability Experiments and Analysis," Japan Atomic Energy Research Institute Report 92-069, May 1992.

9 J.C. DOYLE, K. GLOVER, P.P. KHARGONEKAR, B.A. FRANCIS, "State-Space Solutions to Standard $\mathcal{H}_{2}$ and $\mathcal{H}_{\infty}$ Control Problems," Proc. 19th Symp. Fusion Engineering, Knoxville, Tennessee, October 2-6, 1989, Vol. 34, p. 831, Institute of Electrical and Electronic Engineers.

10 J.C. DOYLE, J.E. WALL, G. STEIN, "Performance and Robustness Analysis for Structured Uncertainty," Institute of Electrical and Electronic Engineers Conf. on Decision and Control, December 1982, p. 629.

11 E.M. KASENALLY, D.J.N. LIMEBEER, and A. PORTONE, "Robust Instability Control of Tokamak Plasmas," Proc. of the IFAC World Congress, Sydney, Australia, Vol. 4, 1993, p 389.

12 M. FIRESTONE, C.E. KESSEL, J. MORROW-JONES, S.C. JARDIN, T.K. MAU, "Comprehensive Control of a Tokamak Reactor," Proc. of 14th Symp. on Fusion Engineering, San Diego, California, September 30 through October 3, 1991, Vol. 1, p. 209. 

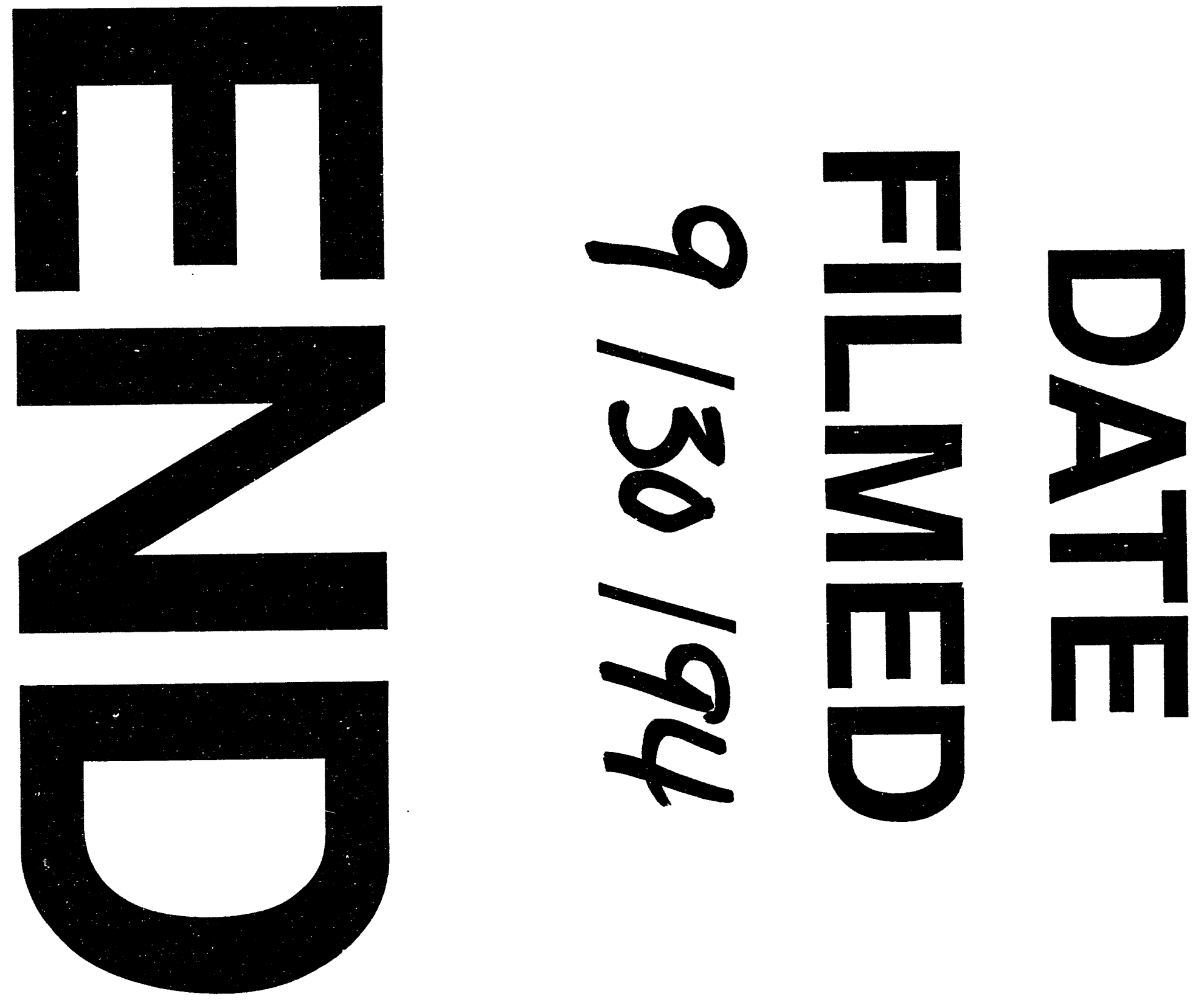